\title{
Modelling of the TH-dependent regulation of tadpole tail resorption.
}

\author{
S. Troncale ${ }^{1}$, R. Thuret ${ }^{2}$, A.-C. Fierro ${ }^{2}$, C. Ben ${ }^{2}$, N. Pollet ${ }^{2}$, J.-P. Comet ${ }^{1,3}$ and G. Bernot ${ }^{3}$
}

May 31, 2007

\author{
${ }^{1}$ IBISC, FRE 2873, Université d'Evry Val- \\ d'Essonne, Génopole, France \\ ${ }^{2}$ Laboratoire de Développement et Evolution, UMR \\ 8080, Université de Paris Sud, France \\ ${ }^{3}$ Programme d'Epigénomique, Génopole et Univer- \\ sité d'Evry, France
}

Short title : Modelling of tail resorption regulation

\begin{abstract}
Tail resorption observed at the time of amphibian metamorphosis is controlled by thyroid hormone. The inherent regulation network is complex and involves an important number of different factors. Consequently, the global understanding of this biological process needs elaborated experiments. However, these experiments can prove to be difficult to realize because of the need to manipulate in space and in time gene expression and hormonal treatments. Hence, we first modelled and simulated this biological process using Hybrid Functional Petri Nets. This powerful formalism offers a number of features and flexibility. Curves obtained in silico by simulations are in agreement with those observed in vivo and in vitro. Our modelling approach led us to ask pertinent biological questions. From these questions, new hypothesis which stay to be experimentally tested have followed.
\end{abstract}

Key words : metamorphosis, tail resorption, thyroid hormone, modelling, Hybrid Functional Petri Nets

\section{Introduction}

Most amphibians undergo numerous morphological changes at the tadpole stage, a biological process called metamorphosis. Amphibian metamorphosis can be divided into three periods. During premetamorphosis the feeding tadpole grows. During prometamorphosis hind limbs grow and differentiate. Finally, tail resorption characterizes metamorphic climax. All these modifications are under control of thyroid hormone [1].

Among all the changes related to metamorphosis, we were particularly interested in the regulatory mechanisms responsible for tail resorption [2]. Indeed, apoptotic mechanisms are triggered [1] and a better understanding of regulations inducing apoptosis could have important therapeutic consequences. However, the experimental analysis of the ongoing regulations is difficult. Indeed, relevant molecules (hormones, proteins) are difficult to observe and manipulate in a spatio-temporal manner in the context of metamorphosis.

Therefore the experimental study of this process is slowed down. A first step of modelling is much more flexible. In silico tests of biological hypothesis are then strongly made easier. In this paper, we construct a model representing kinetics of different factors which interfere in tail resorption of the tadpole Xenopus tropicalis. A formalism offering a maximum of flexibility is required to study this complex biological process implicating a large number of interacting factors. Accordingly, we chose Hybrid Functional Petri Nets (HFPN)) [3, 4], a formalism allowing to model discrete and continuous processes, 
to model consumption and production of resources, and to consider any relations between factors.

The model elaboration was enabled thanks to close collaboration between biologists and modelling specialists. The interaction was productive to ask pertinent questions in a biological system point of view, and to formulate hypothesis on the role of some cells in function of their location in respect to plasmatic flow. Eventually, the kinetics obtained after simulations are in agreement with those listed in the literature.

This paper is organized as follows : the biological context is first presented in section 2, and the HFPN formalism is described in section 3. Construction of our model is detailed in section 4 and our simulation results are exposed in section 5. Section 6 presents an in silico test of an enzymatic overexpression. Finally, we discuss these results as well as the contribution of the interdisciplinarity in the study of biological complex systems.

\section{Biological context}

The whole development of the Xenopus frog, starting from the zygote (fertilized egg) and ending at the adult form, has been described as a succession of discrete stages classified using anatomical traits (Nieuwkoop and Faber [5]; Figure $1 \mathrm{ex}-$ tracted from [6]). Metamorphosis encompass the period covering the stages 54 to 66 (the last stage of development). During metamorphosis, the Xenopus tropicalis tadpole undergoes a series of morphological changes. All metamorphosis-associated morphological changes are under the control of thyroid hormones, denoted TH. It is relevant to distinguish two molecular forms of TH [2]. Thyroxine (tetraiodothyronine, T4) corresponds to the major form secreted by the thyroid gland, it is an "inactive" form of $\mathrm{TH}$ and is considered a pro-hormone. Triiodothyronine (T3) is the biologically active form but it is secreted in a smaller quantity [7]. Complex regulations in which signals emitted by lots of organs, such as pituitary gland or liver, intervene are regulating the synthesis of thyroid hormones. Newly produced TH are secreted in the plasma and supply organs. It results from this regulation a strong increase of plasmatic $\mathrm{TH}$ concentration during prometamorphosis up to a peak at the climax of metamorphosis (stages 6263) [6] (Figure 1).

The TH-dependant regulation at the origin of tail resorption requires a strong intra-cellular $\mathrm{TH}$ concentration [1]. This regulation is triggered at the climax of metamorphosis when plasmatic TH concentration is maximal. Intra-cellular $\mathrm{TH}$ bind their nuclear receptors existing under two forms : TR- $\alpha$ and TR- $\beta[8,1,9]$. The TH/TR complex bound to DNA recruits a co-activator complex that activates expression of downstream genes, ultimately resulting in programmed cell death. On one hand, TR- $\alpha$ is observed in a ubiquitous manner in the tail (Figure 1), it binds its co-factor RXR- $\alpha$ which will not be considered in our model since its ubiquitous character. On the other hand, TR- $\beta$ is a direct-response gene of $\mathrm{TH}$ [1]. Its expression then increases with endogenous TH concentration (Figure 1).

The intra-cellular TH concentration depends on the plasmatic contribution, but it is also regulated by two enzymes, type 2 deiodinase, D2 [10] and type 3 deiodinase D3 [11]. Type 2 deiodinase synthesizes the active form of the hormone (T3) from its inactive form (T4) : D2 $+T 4 \rightarrow T 3$. On the contrary, type 3 deiodinase inactivates the two hormone types T3 and T4:D3 $+T 4 \rightarrow r T 3$ and $D 3+T 3 \rightarrow T 2$, where rT3 and T2 are inactive. The gene expressing D3 is a direct-response gene of TH [11]. D3 concentration increases with this of T3 and, D3 concentration is then down-regulated at the climax of metamorphosis (stages 60-61) [10]. Expression of gene encoding D2 is also regulated by thyroid hormone, but in an indirect manner. D2 concentration then increases with this of $\mathrm{T} 3$, but response time is much longer (few days) [10]. Concentration of this enzyme reaches a peak at the climax of metamorphosis (stages 62-63) [12] and decreases when tail degenerates.

\section{FIGURE 1}




\section{HFPN formalism}

Modelling of tail resorption in the $X$. tropicalis tadpole must integrate notions of consumption and production of resources in a way of observing quantitatively evolution of intra-cellular TH concentration. Moreover, two kinds of biological processes can be distinguished : continuous processes, such as enzymatic activity defined by the Michaelis constant $(\mathrm{Km})$ and by the maximal rate, and discrete processes matching here essentially biological processes activated after a delay of time. We then considered a hybrid modelling allowing consideration of discrete and continuous processes. Hybrid functional Petri nets (HFPN) [3, 4] represent an adequate formalism for hybrid models. The functional aspect of these nets is related to possibility to define consumed or produced quantity as well as reaction rates using functions depending on other factors of the net. For example, if we consider rate of a continuous transition, this can be expressed by function of marking of one or fewer places. The incidence condition between the transition and a place is not necessary to express the rate dependence in function of marking of a place. Then HFPN enable a large freedom in the interaction description, conserving the usual representation of Petri nets [13]. They are composed of places, transitions and arcs being either discrete or continuous (Figure 2) :

- Discrete places represent entities which can be numbered (thanks to tokens).

- Continuous places represent entities which can not be numbered. A real number (representing a concentration for example) is associated with each continuous place.

- Discrete transitions are fired after a delay of time $d t$.

- Continuous transitions are continuously fired at a rate $v(t)$

- Normal arcs activate transitions by consuming resources.

- Inhibitory arcs activate transitions only when a resource is absent.
- Test arcs activate transition without consumption of resources.

\section{FIGURE 2}

\section{Model construction}

Since our goal is to study causalities leading to tail resorption from plasmatic $\mathrm{TH}$ concentration, plasmatic TH concentration curves are then considered as result of regulations upstream of the studied process.

The model presented below imposes curves of $\mathrm{T} 3 \mathrm{p}$ and T4p concentration in agreement with those observed on Figure 1 (see sub-section 4.1 where the function describing hormone evolution is constructed to obtain such curves). These kinetics stand for input data in our model. The model also integrates regulations related to intra-cellular TH, D2, D3, TR- $\beta, \ldots$ Its elaboration is addressed through construction of sub-modules. We begin with role of plasmatic TH.

\subsection{TH plasmatic modelling}

The first step in our model construction was to model plasmatic $\mathrm{TH}$ concentration related to those observed on Figure 1. These curves are used as the model base. Plasmatic T4 and T3 concentration are modelled thanks to two continuous places, called $\mathrm{T} 4 \mathrm{p}$ and T3p on the final model (Figure 3). The continuous transition $t_{1}$ represents continuous synthesis of thyroid hormone by the thyroid gland. When T3p and $\mathrm{T} 4 \mathrm{p}$ respectively reach their peak, the transitions $t_{2}$ and $t_{3}$ provide a continuous token to places $\mathrm{p} 1$ and $\mathrm{p} 2$. This signal models metamorphic climax and plasmatic TH concentration can then decrease.

The resulting sub-model only uses one place and one transition for each hormone. For example, the modelling of plasmatic T4 (T4p) only needs $t_{2}$ and p1. This modelling was made easier thanks to use of functions on arcs and transitions. For example, the arc from the place $\mathrm{T} 4 \mathrm{p}$ to the transition $t_{2}$ possesses a function explaining: If the climax of metamorphosis is not still reached, then plasmatic T4 concentration linearly increases, else this concentration linearly 
declines until a basal value. The use of functions allows to model complex reactions with few places and transitions.

FIGURE 3

\subsection{Intra-cellular TH modelling}

A proportion of plasmatic TH passes in tail cells and contributes to the increase of intra-cellular $\mathrm{TH}$ concentration. The plasmatic T4 crossing is modelled by the continuous transition $t_{4}$ (Figure 3 ) which supplies the cell with intra-cellular T4 (continuous place $\mathrm{T} 4 \mathrm{c}$ ). The same process is used for intracellular T3 (continuous transition $t_{5}$ and continuous place $\mathrm{T} 3 \mathrm{c})$.

\subsection{D2 role modelling}

When the intra-cellular $\mathrm{T} 3$ concentration reaches a threshold, T3 activates intermediate genes [12], which then induce D2 expression, initially absent in the tadpole tail [10]. Consequently, a time delay exists between the threshold reached by $\mathrm{T} 3 \mathrm{c}$ and the observation of D2 in the tail. This time delay is modelled by the discrete transition $t_{6}$ (Figure 3 ). Once the time is elapsed, intermediate genes (continuous place GI) cause D2 concentration increase (continuous place D2). The active form of thyroid hormone (T3c) is synthesized by D2 from the inactive form (T4c) (continuous transition $t_{8}$ ). Since D2 is not consumed at the moment of reaction, a test arc is used.

\subsection{D3 role modelling}

Contrarily to D2, D3 is initially present in the tadpole tail (continuous place D3) [11]. D3 concentration is directly regulated by the intra-cellular T3 (T3c). This activation is modelled by a continuous transition $t_{9}$ (Figure 3). Since T3c concentration does not decrease at the moment of this regulation, a test arc is used. Type 3 deiodinase inactivates the two hormone kinds, T3 and T4. This inhibition is modelled by the continuous transition $t_{10}$, which decreases $\mathrm{T} 3 \mathrm{c}$ and $\mathrm{T} 4 \mathrm{c}$ concentration when it is fired. Functions were also integrated so that the enzymatic reaction is proportional to the resource quantity (D3 and $\mathrm{TH}$ ).

\section{$4.5 \quad$ TR- $\beta$ role modelling}

One of the thyroid hormone receptors, TR- $\alpha$ is ubiquitously present in tail cells, while TR- $\beta$ is a direct-response gene of TH $[1,8]$. A hypothesis considers TR- $\alpha$ as a transcription factor of early genes and TR- $\beta$ as a transcription factor of late genes leading to apoptosis [1]. Since the ubiquitous character of TR- $\alpha$, this is not explicitly represented in our model. When $\mathrm{T} 3 \mathrm{c}$ reaches a threshold, it activates early gene transcription, among which we distinguish TR- $\beta$. This continuous process is modelled by the transition $t_{11}$ (Figure 3), which fills continuous places GP and TR- $\beta$ respectively associated to early genes and to TR- $\beta$. This transition is only fired when the threshold indicated on arc is reached. Finally, it is strongly probable that one of the early genes is responsible for D3 inhibition [11]. When early genes are activated (place GP filled), the continuous transition $t_{12}$ is activated its turn, decreasing D3 concentration.

\subsection{Apoptosis activation modelling}

Once TR- $\beta$ is activated, it forms a complex with T3c. The transcription factor well formed enables the induction of late apoptotic genes (place GA on Figure 3 ). This reaction can only occur when a strong $\mathrm{T} 3 \mathrm{c}$ concentration is present in the cell during a delay of time [1]. This delay is modelled by the discrete transition $t_{13}$. The threshold enabling activation of this transition is more important than this previously cited. Early gene expression then occurs before late gene expression. To finish, among apoptotic genes there are proteases which damage still present proteins, that is to say D2 and TR- $\beta$. This process is modelled by the transitions $t_{14}$ and $t_{15}$.

\section{Results}

The Cell Illustrator software [3, 4], available at the web site http://www.GenomicObject.Net/, implements HFPN formalism and allows us to test our 
model through a run of simulations. We observed concentration evolution of the following elements in function of time : plasmatic T3 and T4 (T3p and $\mathrm{T} 4 \mathrm{p}$ ), intra-cellular T3 and T4 (T3c and T4c), D2, D3, TR- $\beta$ and the expression of apoptotic gene (GA). Results are presented on the Figure 4.

\section{FIGURE 4}

In a qualitative point of view, curves observed in silico are in agreement with kinetics (expressed in arbitrary unit) observed in vivo and in vitro $[6,1$, $10,11,12]$. As a matter of course, T3p and T4p curves (piece linear) abstract the behavior resulting of upstream regulations (see sub-section 4.1). While D3 is present in cell, intra-cellular TH concentration stays low. It then increases when type 3 deiodinase is down-regulated. In vivo, D3 inhibition marks D2 activation. This activation is observed in silico at time 5.0 of our simulation. D2 presence enables to obtain very quickly a concentration of intra-cellular T3 which is higher than the intra-cellular T4 concentration.

The inductions of genes expressing TR- $\beta$ and D3 are similar. Nevertheless, contrarily to D3, TR- $\beta$ is only down-regulated when tail degenerates (time 8.0 on the Figure 4). Finally, when the intra-cellular T3 concentration reaches a threshold, the transcription factor T3/TR- $\beta$ enables induction of apoptotic gene expression. Apoptosis trigger is observed with the increase of the apoptotic gene concentration. Let note that a development with stage is not biologically significant : this curve has a similar role as a button switch, it indicates if apoptosis is triggered or not.

\section{D3 overexpression}

So as to validate our model in a informal manner, a test which has already been performed in vivo was tested in silico. A tadpole overexpressing deiodinase of type 3 was simulated. In vivo these tadpoles were obtained by usual protocols of transgenesis [11]. Consequence of such an experiment is the ubiquitous expression of D3 in tail cells, D3 concentration is then strong during all the metamorphosis. In silico, this process was modelled by removing transitions responsible for D3 regulation. Then transitions $t_{9}$ and $t_{12}$ were removed from the final model (Figure 3) and the initial value of D3 marking was also increased in order to model overexpression. Simulations led to results presented in Figure 5.

\section{FIGURE 5}

$\mathrm{T} 3 \mathrm{p}$ and $\mathrm{T} 4 \mathrm{p}$ curves are unchanged in respect to previously. T3c and T4c concentrations stay low all along the metamorphosis. Curves show that $\mathrm{T} 3 \mathrm{c}$ and $\mathrm{T} 4 \mathrm{c}$ do not succeed to reach threshold leading to expression of D2 or TR- $\beta$. Consequently, apoptotic genes are never induced. The tail death program is then not set in motion. We can conclude that the tadpole modelled in silico keeps its tail despite of metamorphosis.

Such in vivo experiments are hard to perform. Indeed, most of the animals do not survive. A single tadpole reached the adult age and this animal keept its tadpole tail [11].

This in silico test then allows a informal validation of our model.

\section{Discussion}

In this paper, a model simulating the $\mathrm{TH}$ dependent regulation responsible for tail resorption in the Xenopus tropicalis tadpole is developed. The obtained model provides results in agreement with those notified in the literature. Modelling of this biological process required the use of an expressive formalism. We concentrated on the HFPN formalism which enable modelling of discrete and continuous processes as well as modelling of consumption and production of resources. This formalism has the distinctiveness to make easier modelling of complex regulations thanks to the use of functions on arcs and transitions. Despite of expressiveness of the HFPN formalism, it would be interesting to compare and precisely evaluate contributions and limits of the HFPN formalism in respect to the other formalisms used in biology.

The main goal of a modelling work is to lead to a better understanding of the biological system. In our case, the model was constructed in the goal of going into detail our knowledge about specific functionali- 
ties of thyroid hormone in the tail. The initial question refers to the TH nature: can we talk about morphogen for the thyroid hormone? A morphogen is a secreted molecule which has the property to induce different cellular types with different concentrations. Once the model was constructed, in silico tests led us towards an in-depth study of the role and activities of type 2 and 3 deiodinases. The kinetics data (Km and Vmax) given by Germain et al. in ?? show that D2 is 10 times less active than D3. Moreover, since plasma supplies single-handedly a strong T3 concentration, we question us on matter of D2 role. Intermediate models were then developed to simulate competition between D2 and D3. We first suggested that our regulation network presented in this paper was mutual to each tail cell. Nevertheless, simulation results contradicted this hypothesis and new questions were then asked: which cells express D2 and D3 in the tadpole tail, do some cells express D2 and do the other express D3,... A bibliographical study demonstrated that deiodinases are exclusively expressed in a minority group of tail cells $[10,9]$. Cells expressing these enzymes are surprisingly those that are the most vascularized, and consequently those receiving a maximum of plasmatic TH. At the opposite, cells that are the least vascularized do not express D2. We can then ask us how these cells undergo apoptosis. Two hypothesis are conceivable :

- Cells expressing D2 could be used as spatial relay towards cells which do not express the enzyme. The most vascularized cells would accumulate a strong T3 concentration, which would then diffuse towards cells lacking of this hormone. This spatial relay would also be temporal because it would allow the maintain of a strong T3 concentration in cells expressing D2 in despite of the decline observed in the plasma, which does not maintain the regular T3 supply.

- The second hypothesis considers two types of death in tail. Cells expressing D2 would die by apoptosis, while death of the other cells would be the consequence of apoptosis of the first cell class. Some papers already identified several resorption programs [14, 9], but none correlation with D2 expression has been done.
These two hypothesis are not in contradiction and could then coexist in a same regulation system. Currently, we work on elaboration of experimental validation methods enabling the confirmation or the invalidation of these hypothesis.

This model fits only the first step in the understanding of the studied biological system. This first step was performed using numeric values of the different parameters which are consistent with published knowledge. In the following we envisage a work on in silico parameter estimation (slot of consistent parameters, stability, robustness...). We also envisage to add new factors, such as corticosteroids, which enable D3 inhibition and D2 activation. The model enrichment will enable to connect our specific model with surrounding biological processes.

\section{References}

[1] Z. Wang and D. Brown. Thyroid hormoneinduced gene expression program for amphibian tail resorption. Journal of Biological Chemistry, 268:16270-16278, 1993.

[2] C. Rose. Integrating ecology and developmental biology to explain the timing of frog metamorphosis. TRENDS in Ecolgy and Evolution, 20:129-135, 2005.

[3] H. Matsuno, Y. Tanaka, H. Aoshima, A. Doi, M. Matsui, and S. Miyano. Biopathways representation and simulation on hybrid functional Petri nets. In Silico Biology, 3:389-404, 2003.

[4] A. Doi, S. Fujita, H. Matsuno, M. Nagasaki, and S. Miyano. Constructing biological pathway models with hybrid functional Petri nets. In Silico Biology, 4:271-291, 2004.

[5] P.D. Nieuwkoop and J. Faber. Normal Table of Xenopus laevis. Garland Publishing Inc, 1956.

[6] J. Leloup and M. Buscaglia. Triiodothyronine, hormone of amphibian metamorphosis. C.R. Acad. Sci., pages 2261-2263, 1977.

[7] J.D. Furlow and E.S. Neff. A developmental switch induced by thyroid hormone: Xenopus 
laevis metamorphosis. TRENDS in Endocrinology and Metabolism, 17:40-47, 2006.

[8] A. Kawahara, B. Baker, and J.-R. Tata. Developmental and regional expression of thyroid hormone receptor genes during Xenopus metamorphosis. Development, 112:933-943, 1991.

[9] D. Berry, R. Schwartzman, and D. Brown. The expression pattern of thyroid hormone response genes in the tadpole tail identifies multiple resorption programs. Developmental Biology, 203:12-23, 1998.

[10] L. Cai and D. Brown. Expression of type 2 iodothyronine deiodinase marks the time that a tissue responds to thyroid hormone-induced metamorphosis in Xenopus laevis. Developmental Biology, 266:87-95, 2003.

[11] H. Huang, N. Marsh-Armstrong, and D. Brown. Metamorphosis is inhibited in transgenic Xenopus laevis tadpoles that overexpress type 3 deiodinase. Proc. Nat. Acad. Sci., 96:962-967, 1999.

[12] H. Huang, L. Cai, B. Remo, and D. Brown. Timing of metamorphosis and the onset of the negative feedback loop between the thyroid gland and the pituitary is controlled by type 2 iodothyronine deiodinase in Xenopus laevis. Proc. Nat. Acad. Sci., 98:7348-7353, 2001.

[13] R. David and H. Alla. Discrete, Continuous, and Hybrid Petri Nets. Springer, 2005.

[14] R. Elinson, B. Remo, and D. Brown. Novel structural elements identified during tail resorption in Xenopus laevis metamorphosis: Lessons from tailed frogs. Developmental Biology, 215:243-252, 1999. 

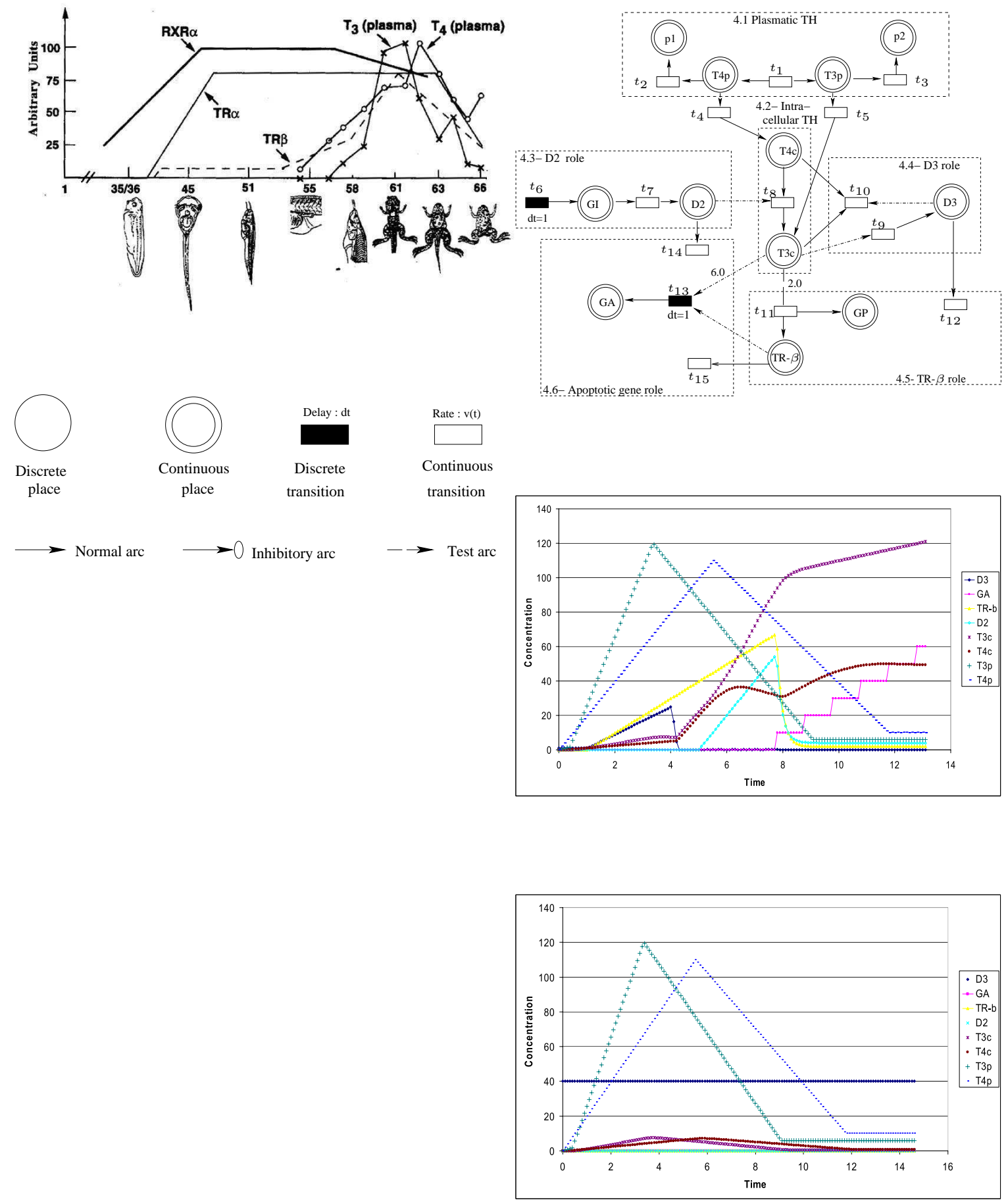
Figure 1: Correlations between levels of endogenous TH and mRNA, of TR- $\alpha$, TR- $\beta$ and RXR- $\alpha$ genes during the different development stages. Figure extracted from [6].

Figure 2: Representation of the different HFPN components.

Figure 3: Final model representing interactions between plasmatic TH (T4p and T3p), intra-cellular TH (T4c and T3c), deiodinases of type 2 and type 3 (D2 and D3) and the TH receptor TR- $\beta$. Intermediate genes (GI), early genes (GP) and apoptotic late genes (GA) are also represented.

Figure 4: Concentration evolution of the different factors (simulations made by Cell Illustrator)

Figure 5: Concentration evolution of the different factors when D3 is overexpressed (simulations made by Cell Illustrator) 